\title{
Aging Measurements with the Gas Electron Multiplier (GEM)
}

\author{
M.C. Altunbas ${ }^{\mathrm{a}}$, K. Dehmelt ${ }^{\mathrm{b}}$ S. Kappler ${ }^{\mathrm{cd} *}$, B. Ketzer ${ }^{\mathrm{c}}$, L. Ropelewski ${ }^{\mathrm{c}}$, F. Sauli ${ }^{\mathrm{c}}$, F. Simon ${ }^{\mathrm{e}}$ \\ a State University of New York, Buffalo, U.S.A. \\ ${ }^{\mathrm{b}}$ Institut für Kernphysik, Mainz University, Germany \\ ${ }^{\mathrm{c}}$ CERN, EP division, CH-1211 Geneva 23, Switzerland \\ dInstitut für Experimentelle Kernphysik, Karlsruhe University, Germany \\ e Department E18, Munich Technical University, Germany
}

Continuing previous aging measurements with detectors based on the Gas Electron Multiplier $($ GEM), a $31 \times$ $31 \mathrm{~cm}^{2}$ triple-GEM detector, as used in the small area tracking of the COMPASS experiment at CERN, was investigated. With a detector identical to those installed in the experiment, long-term, high-rate exposures to $8.9 \mathrm{keV} \mathrm{X}$-ray radiation were performed to study its aging properties. In standard operation conditions, with Ar: $\mathrm{CO}_{2}$ (70:30) filling and operated at an effective gain of $8.5 \cdot 10^{3}$, no change in gain and energy resolution is observed after collecting a total charge of $7 \mathrm{mC} / \mathrm{mm}^{2}$, corresponding to seven years of normal operation.

This observation confirms previous results demonstrating the relative insensitivity of GEM detectors to aging, even when manufactured with common materials.

Keywords: Gas Electron Multiplier; GEM; high rate radiation detector; aging measurements

\section{Introduction}

The Gas Electron Multiplier (GEM) [1] is a two-side copper-clad Kapton foil, perforated with a high density of holes. Etched in a photolithographic process, these holes have diameters of about $70 \mu \mathrm{m}$ and are placed with a pitch of usually $140 \mu \mathrm{m}$ (standard geometry), as shown in figure 1 .

Application of a potential difference between upper and lower copper layer creates a high electric field inside the holes. Figure 2 illustrates, that inserted in the drift gap of a gas detector, nearly all drifting electrons are guided into these holes, where they undergo proportional gas amplification. A large fraction of the electrons is released into the volume below the GEM, resulting in effective gains well above $10^{3}$.

Stacking several GEMs in a cascade leads to the multi-GEM detector. The total effective gain obtained with such a detector can be adapted to the needs of the application choosing the number of

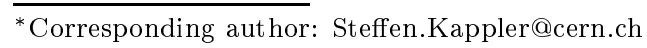

GEMs and the voltage across each one. The major advantage of the GEM detector is the separation of gas amplification and readout stage. This separation results in a fast readout signal (only electrons are collected by the readout structure), and provides a margin of safety in case of discharges occurring in the GEM foils. Furthermore, it also allows high flexibility in the geometry of the readout structure so that a two-dimensional detector readout becomes easily realizable.

\section{Previous aging measurements}

As already demonstrated in previous measurements [2], GEM detectors seem a priori less sensitive to aging than other micropattern devices. Figure 3, for instance, shows the result of measurements with a small double-GEM detector in Ar- $\mathrm{CO}_{2}$ (70:30). There, the two standard geometry GEMs were glued to frames (G10) and stacked between a drift cathode of aluminized Mylar and a one-dimensional strip readout inside a fibreglass (Stesalit) box. A rubber O-ring guaranteed gas- 


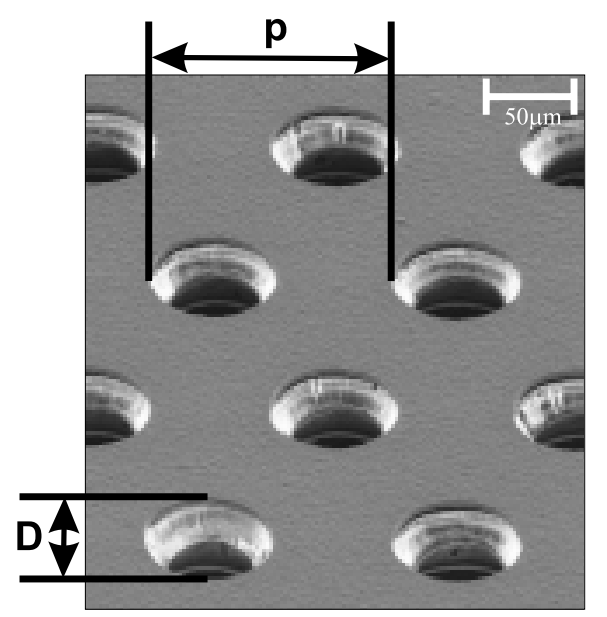

Figure 1. Electron microscope photograph of a GEM foil with standard geometry $(p=140 \mu \mathrm{m}$ pitch and $D=70 \mu \mathrm{m}$ outer hole diameter).

tightness between box and top frame that has a thin window. With GEM voltages of $410 \mathrm{~V}$ each, electric fields of $2.0 \mathrm{kV} / \mathrm{cm}$ in the drift gap, and $4.0 \mathrm{kV} / \mathrm{cm}$ in the other gaps, an effective gain of $2.2 \cdot 10^{3}$ was achieved. On a spot of $2 \mathrm{~cm}^{2}$, the detector was irradiated with $6 \mathrm{keV} \mathrm{X}$-rays at a rate of $4.75 \cdot 10^{4} \mathrm{~Hz} / \mathrm{mm}^{2}$, resulting in a current density of $3.7 \mathrm{nA} / \mathrm{mm}^{2}$. A small single wire proportional counter (SWPC) was installed in the same gas line, behind the GEM detector. After a total collected charge of $12 \mathrm{mC} / \mathrm{mm}^{2}$, the GEM detector did not show any loss in gain, whereas the SWPC did. This might be explained by the presence of outgassing or polymerization products, which did not harm the GEM detector, but triggered aging in the SWPC.

As a necessary complement to these measurements performed with small detectors and under somewhat ideal conditions, a triple-GEM detector from the production line of the COMPASS experiment was investigated as described in the following sections.

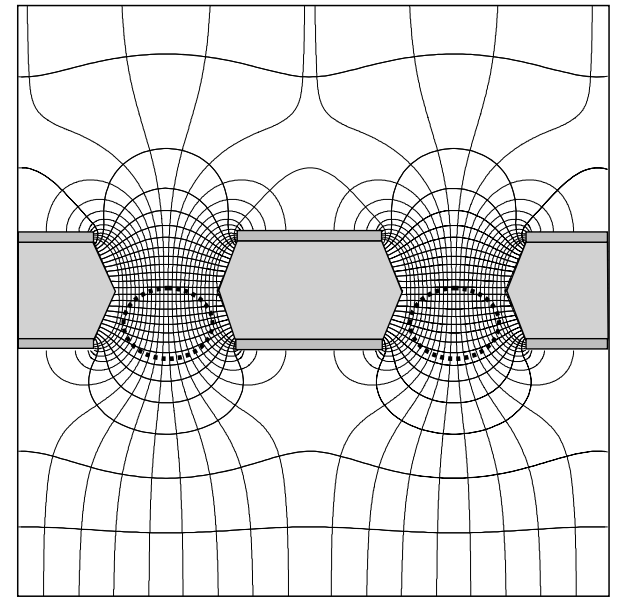

Figure 2. Electric field lines in a GEM foil. The dotted ring indicates the region of highest charge density.

\section{The COMPASS triple-GEM detectors}

The COMPASS experiment (COmmon Muon and Proton Apparatus for Structure and Spectroscopy) is a fixed target experiment at CERN's Super Proton Synchrotron (SPS), built for the investigation of hadron structure and hadron spectroscopy using high intensity muon and hadron beams [3]. In its final setup, this experiment will use 20 triple-GEM detector modules with an active area of $31 \times 31 \mathrm{~cm}^{2}$ for small area tracking.

The design of the COMPASS GEM detectors was optimized to satisfy the low material budget requirement of the experiment while achieving good space and time resolution properties.

In order to minimize probability and energy of spark discharges, the gas amplification is distributed to three GEM foils, which are on one side segmented into 12 sectors plus a central disk, and powered by a resistive voltage divider. At nominal operation, the three GEMs have a potential difference of $425 \mathrm{~V}$ on the first, $380 \mathrm{~V}$ on the middle, and $340 \mathrm{~V}$ on the third GEM. The electric fields are chosen to $2.5 \mathrm{kV} / \mathrm{cm}$ in the drift gap and $3.7 \mathrm{kV} / \mathrm{cm}$ in all other gaps. By lowering the 


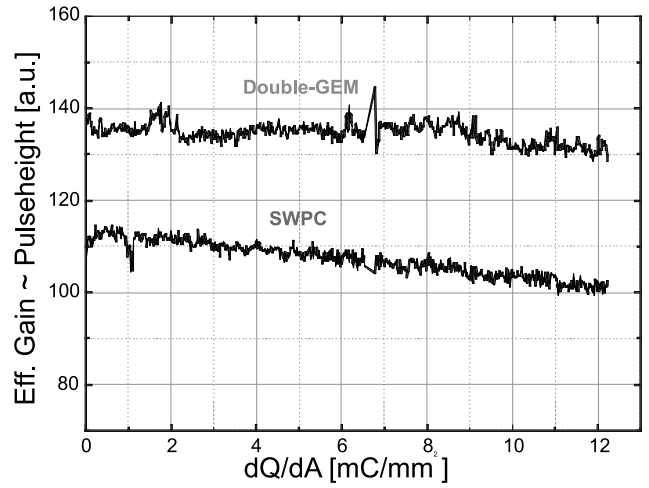

Figure 3. Previous aging measurement of a double-GEM detector with $\mathrm{Ar}-\mathrm{CO}_{2}$ (70:30): effective gain versus accumulated charge $d Q / d A$.

voltage on the central disk sector of the lowest GEM, this region can be deactivated to allow operation with the high intensity beam traversing the detector volume.

The detectors are equipped with a twodimensional projective strip readout, consisting of two layers of 768 strips at $400 \mu \mathrm{m}$ pitch $(70 \mu \mathrm{m}$ width in the upper, $350 \mu \mathrm{m}$ in the lower layer), engraved on the two sides of a $50 \mu \mathrm{m}$ Kapton foil. With the Kapton removed in the interstecies of the upper strips, both layers are opened for charge collection (see figure 4).

As shown in the exploded view in figure 5, the strip readout is glued to a $3 \mathrm{~mm}$ thick carrier structure made of honeycomb, which is covered by $125 \mu \mathrm{m}$ Stesalit skins on each side. The GEMs are glued to three fibreglass (Vetronite) frames of $2 \mathrm{~mm}$ thickness, which also contain spacing grids to maintain the gap width constant over the whole area. To avoid possible charging up effects or discharges close to these grids, their surface is smoothed with a thin layer of Polyurethane. The last frame is made of $3 \mathrm{~mm}$ thick Stesalit, and carries the drift cathode, consisting of a $50 \mu \mathrm{m}$ thick Kapton foil with $5 \mu \mathrm{m}$ copper. For stability reasons, the whole structure is closed by another $3 \mathrm{~mm}$ carrier honeycomb, resulting in a total

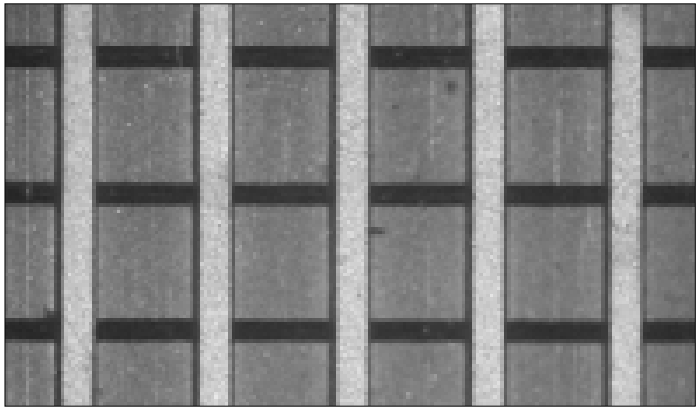

Figure 4. Microscope photograph of the strip readout. (Pitch $400 \mu \mathrm{m}$, strip width $70 \mu \mathrm{m}$ in the upper and $350 \mu \mathrm{m}$ in the lower layer.)

module thickness of $15 \mathrm{~mm}$ at a radiation length of $7.19^{\circ} /$ oo only [4]. The counting gas is supplied through $0.5 \mathrm{~m}$ long Polypropylene tubes and distributed via grooves machined into the uppermost frame. The component assembly was done with the non-outgassing glue Araldit AY103 plus hardener HD991 [6]. For completion, all components and materials used for detector production are listed in table 1; a more detailed description of the detector construction can be found in [5].

Operated in $\mathrm{Ar}-\mathrm{CO}_{2}$ (70:30) at an effective gain of $8.5 \cdot 10^{3}$, this detector achieves a spatial resolution of $45 \mu \mathrm{m}$ for minimal ionizing particles (MIPs) and a time resolution of $15 \mathrm{~ns}$ [5]. Thus, with a total efficiency of more than $98 \%$ and stable operation even at highest beam intensities, all requirements of the experiment are met.

\section{Aging study setup}

To investigate aging properties, the setup shown in figure 6 was used to irradiate the GEM detector with a high-rate X-ray beam. A small SWPC for reference measurements was installed in the same gas line in front of the GEM detector. Charge sensitive amplifiers were used to regularly record low rate pulse height spectra of both coordinates of the GEM detector and the SWPC. Furthermore, the currents in the GEM detector 


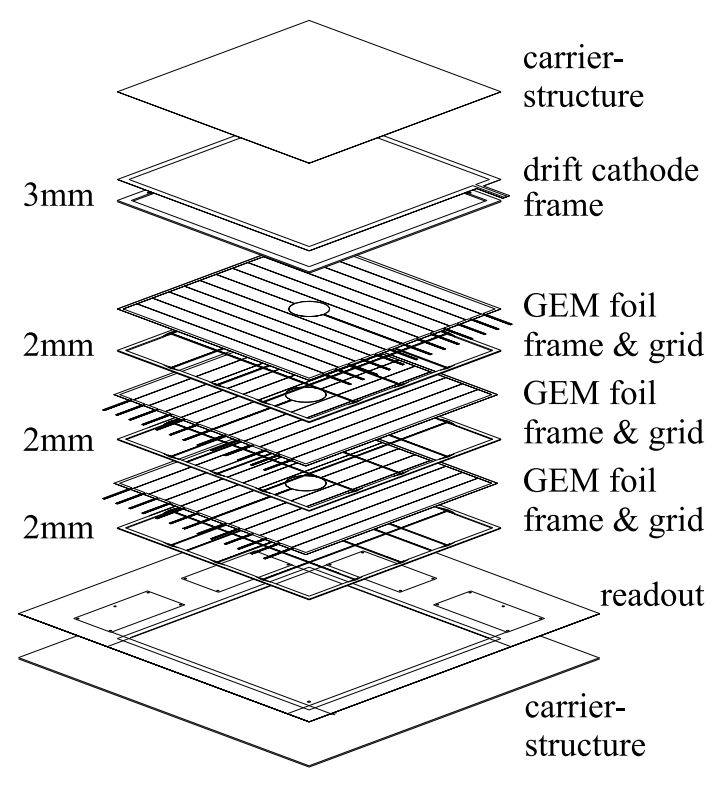

Figure 5. Exploded view of the COMPASS tripleGEM detector design.

and the rate of the X-ray tube were monitored, and sensors for ambient parameters like temperature, relative humidity, and atmospheric pressure were read out.

As in the COMPASS experiment, the gas was supplied with an open flow system. A gas bottle with $\mathrm{Ar}-\mathrm{CO}_{2}$ premixed in a 70:30 ratio was connected via a pressure reducer and a $10 \mathrm{~m}$ long stainless steel gas line to a mass flow control unit. The constant flow of $80 \mathrm{cc} / \mathrm{min}$ was fed to the SWPC and the GEM detector with short Polypropylene tubes. The GEM detector was directly connected to a hygrometer and a $10 \mathrm{~m}$ plastic exhaust gas line, avoiding the use of any bubbler. The detector volume of 0.85 liters was exchanged 5.6 times per hour, somewhat more frequent than in the experiment, where we have 3.5 volume exchanges per hour and two detectors in series per gas line.

One quarter of the detector $\left(15 \times 15 \mathrm{~cm}^{2}\right)$ was exposed to the $8.9 \mathrm{keV} \mathrm{X}$-ray beam with an approximately Gaussian profile of $\sigma=20 \mathrm{~mm}$, thus

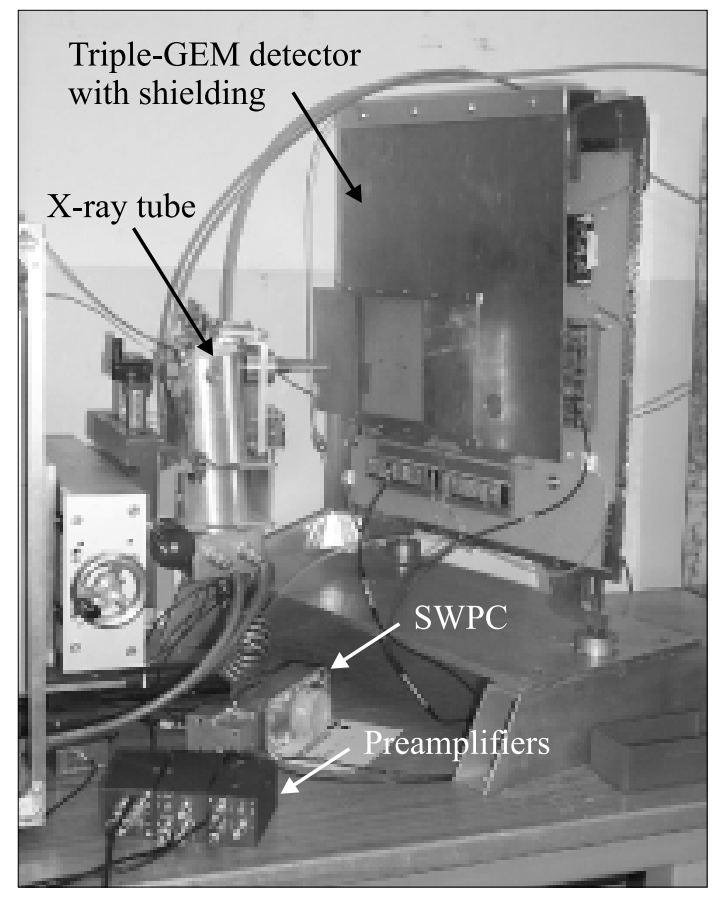

Figure 6. Photograph of the aging measurement setup: X-ray tube and triple-GEM detector with shielding.

with a surface of $12.6 \mathrm{~cm}^{2}$ inside the one- $\sigma$-line. The detector was irradiated in two phases: 10 days at $6.3 \cdot 10^{3} \mathrm{~Hz} / \mathrm{mm}^{2}$ in the beam center, and 10 days at $2.5 \cdot 10^{4} \mathrm{~Hz} / \mathrm{mm}^{2}$. Operated at the nominal effective gain of $8.5 \cdot 10^{3}$, this resulted in a current density of $2.5 \mathrm{nA} / \mathrm{mm}^{2}$ and $10 \mathrm{nA} / \mathrm{mm}^{2}$, respectively. During the whole irradiation, the following measurement cycle was performed: after each ten minutes of irradiation, a shutter in front of the X-ray tube was closed, the low rate pulse height spectra as well as all other parameters recorded, and after reopening the shutter the irradiation continued. In order to measure effective gain $G$ and energy resolution, a Gaussian function was fitted to the pulse height spectra, and position and full-width-half-maximum of the photopeak determined. 


\begin{tabular}{|c|c|c|}
\hline Material & Details & Producer / Supplier \\
\hline \hline Assembly glue & ARALDIT AY103 + HD991 (ratio 10:4) & CERN store \\
\hline Grid conditioning & $\begin{array}{c}\text { NUVOVERN LW } \\
\text { (2 component Polyurethane) }\end{array}$ & $\begin{array}{c}\text { Walter Mader AG, } \\
\text { CH-8956 Killwangen }\end{array}$ \\
\hline Carrier structure & $\begin{array}{c}2 \times 125 \mu \mathrm{m} \text { STESALIT } \\
\text { on } 3 \mathrm{~mm} \text { NOMEX honeycomb }\end{array}$ & $\begin{array}{c}\text { Stesalit AG, CH-4234 Zullwil } \\
\text { Socol, CH-1020 Renens }\end{array}$ \\
\hline Shielding & Aluminium $(10 \mu \mathrm{m})$ & CERN store \\
\hline GEM foils & $2 \times 5 \mu \mathrm{m}$ Cu on $50 \mu \mathrm{m}$ KAPTON & CERN/EST \\
\hline Drift & $5 \mu \mathrm{m}$ Cu on $50 \mu \mathrm{m}$ KAPTON & CERN/EST \\
\hline Frame & $3 \mathrm{~mm}$ STESALIT & CERN/EP/TA1 \\
\hline Spacers & $2 \mathrm{~mm}$ VETRONITE grids & CERN/EST \\
\hline Gas pipes & PP tube $(3 \mathrm{~mm}$ diam. $)$ & Angst-Pfister, CH-8052 Zürich \\
\hline Gas outlet & F-glass + fitting & CERN/EP/TA1 \\
\hline Microstrip readout & Cu microstrips on $50 \mu$ m KAPTON & CERN/EST \\
\hline HV boards & Custom made & CERN/EST \\
\hline
\end{tabular}

Table 1

List of components and materials used for the production of the COMPASS triple-GEM detectors.

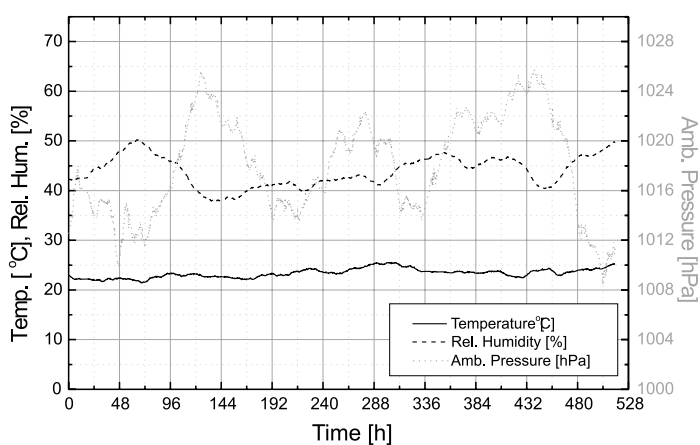

Figure 7. Ambient parameters versus time. (The accuracy is $\pm 1^{\circ} \mathrm{C}$ for temperature, $\pm 3 \%$ for relative humidity, and $\pm 0.5 \mathrm{hPa}$ for atmospheric pressure.)

\subsection{Results}

The variations of the ambient parameters during the aging measurement are plotted in figure 7 . Whereas the relative humidity of the atmosphere varied between $37.9 \%$ and $50.2 \%$, the $\mathrm{H}_{2} \mathrm{O}$ concentration in the counting gas measured with the hygrometer at the output of the detector was con- stant within $2 \mathrm{ppm}$ at $60 \mathrm{ppm}$. The crucial importance of the $\mathrm{H}_{2} \mathrm{O}$ concentration for the discharge probability in GEM detectors has been demonstrated recently [5]: with a double-GEM detector, a change from $81 \mathrm{ppm}$ down to $35 \mathrm{ppm}$ lowered the discharge probability by almost three orders of magnitude (at a gain of $8.5 \cdot 10^{3}$ ). During the aging measurement the currents on the high voltage power supply were monitored and not a single discharge has been observed.

The influence of the variations of temperature $\left(21.4^{\circ} \mathrm{C}\right.$ to $25.5^{\circ} \mathrm{C}$, thus $\left.1.4 \%\right)$ and pressure (1008.4hPa to $1025.7 \mathrm{hPa}$, thus $1.7 \%$ ) on the gas gain are not negligible and had to be considered. Therefore, a corrected and normalized gain $g$ was computed from the effective gain $G$ according to

$g_{i}:=\frac{G_{i}}{A_{i} \cdot e^{B_{i} \cdot T / p}}$,

where $i$ is an index for the two coordinates of the GEM detector and the SWPC and $T / p$ the ratio of temperature and pressure. $A_{i}$ and $B_{i}$ are fit parameters, determined by fitting the exponential function

$G_{i}(T / p)=A_{i} \cdot e^{B_{i} \cdot T / p}$

to the correlation plots of figure 8 (in order not to bias the aging study, only points from the begin- 


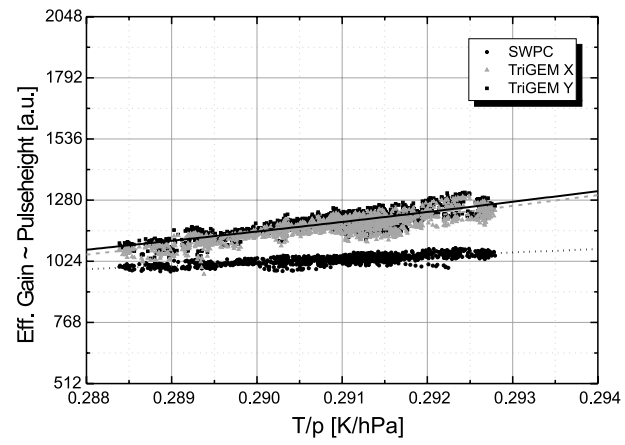

Figure 8. Correlation of detector gain and temperature \& pressure: pulse height versus $T / p$ and the exponential fits.

ning of the measurement were used). The exponential dependence in equation (2) is deduced by assuming inverse proportionality of the Townsend coefficient $\alpha$ to the mass density $\rho$, and thus $\alpha \propto 1 / \rho \propto T / p$.

The results of the aging measurement are given in figure 9. The accumulated charge $d Q / d A$ is given for the one- $\sigma$-line of the beam profile, thus the charge collected in the center was higher by a factor 1.65. One clearly recognizes smaller fluctuations of $7 \%$ in the normalized corrected gain of the GEM detector, completely in phase with the SWPC, and without any reduction of the gain. Apart from small fluctuations, also the energy resolution was constant in each of the two irradiation phases, the improved energy resolution at the beginning of the second irradiation phase is only due to a higher signal to noise ratio. Indeed, in order to compensate the rate-induced voltage drop in the GEM foils, the voltage on the resistive voltage divider was slightly increased, resulting in higher GEM voltages and thus larger signals during the low rate recording phase of the spectra.

After accumulating $7.1 \mathrm{mC} / \mathrm{mm}^{2}$ on the one- $\sigma$ line $\left(11.7 \mathrm{mC} / \mathrm{mm}^{2}\right.$ in the center), the measurement was stopped and a final scan carried out: pulse height spectra were recorded in nine different positions, one in the center of the beam pro-

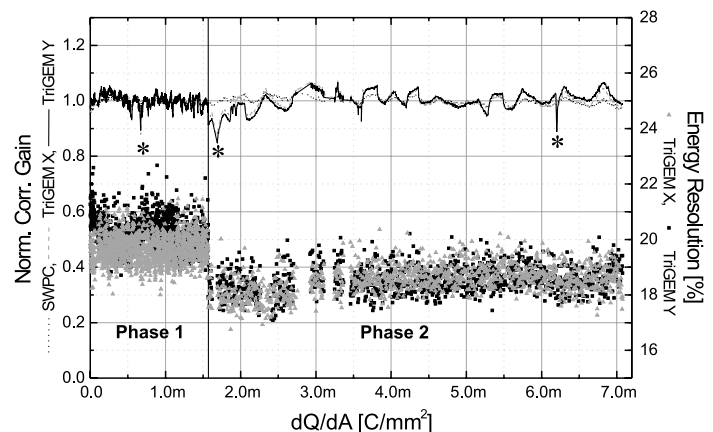

Figure 9. Normalized corrected gain and energy resolution versus accumulated charge $d Q / d A$. The spikes marked with a star $(*)$ indicate an exchange of the gas bottle.

file, four at $19.2 \mathrm{~mm}$, and four at $27.2 \mathrm{~mm}$ distance from the center. All spectra were unchanged, without any loss of gain and energy resolution.

\section{Conclusions}

The aging properties of the triple-GEM detectors built for the COMPASS experiment at CERN were investigated. These low-mass detectors with an active area of $31 \times 31 \mathrm{~cm}^{2}$ are built with a choice of materials imposed by practical constraints, but making use of non-outgassing epoxies [6]. Operated with $\mathrm{Ar}-\mathrm{CO}_{2}$ (70:30) at an effective gain of $8.5 \cdot 10^{3}$, one quarter of the detector area was exposed to an $8.9 \mathrm{keV}$ X-ray beam. After accumulating more than $7 \mathrm{mC} / \mathrm{mm}^{2}$, which corresponds to $1.7 \cdot 10^{11} \mathrm{MIPs} / \mathrm{mm}^{2}$ or seven years of normal operation in COMPASS, no loss of gain and energy resolution was observed.

This observation confirms the results of previous measurements demonstrating the relative insensitivity of GEM detectors to aging when operated with clean $\mathrm{Ar}-\mathrm{CO}_{2}$. Two reasons may explain this result: the fact, that the gas amplification is localized inside the GEM holes (far from any electrodes and walls), and the small effect of possible polymerization deposits on the electric field in this region. 


\section{REFERENCES}

1. F. Sauli, Nucl. Instr. and Meth. A386 (1997) 531.

2. S. Bachmann et al., Development and test of large size GEM detectors, IEEE Trans. Nucl. Sci. NS-47 (2000) 1412.

3. The COMPASS Collaboration, COMPASS: A proposal for a COmmon Muon and Proton Apparatus for Structure and Spectroscopy, CERN/SPSLC 96-14, SPSC/P 297 (1996).

4. B. Ketzer et al., GEM detectors for COMPASS, Paper presented at the IEEE Nuclear Science Symposium and Medical Imaging Conference, Lyon, September 2000.

5. M.C. Altunbas et al., Construction, test and comissioning of the triple-GEM tracking detectors for COMPASS, paper in preparation, to be submitted to Nucl. Instr. and Meth. A.

6. R. Bouclier et al., Effects of outgassing from some materials on gas chamber aging, Nucl. Instr. and Meth. A350 (1994) 464. 